ARTICLE

DOI: $10.1038 / s 41467-018-03935-0$

\title{
Atomically thin noble metal dichalcogenide: a broadband mid-infrared semiconductor
}

\author{
Xuechao Yu (1) 1, Peng Yu², Di Wu ${ }^{3,4}$, Bahadur Singh (10 3,4, Qingsheng Zeng ${ }^{2}$, Hsin Lin ${ }^{3,4}$, Wu Zhou ${ }^{5}$, \\ Junhao Lin (10 ${ }^{6}$, Kazu Suenaga ${ }^{6}$, Zheng Liu (i) ${ }^{2,7} \&$ Qi Jie Wang (i] ${ }^{1}$
}

The interest in mid-infrared technologies surrounds plenty of important optoelectronic applications ranging from optical communications, biomedical imaging to night vision cameras, and so on. Although narrow bandgap semiconductors, such as Mercury Cadmium Telluride and Indium Antimonide, and quantum superlattices based on inter-subband transitions in wide bandgap semiconductors, have been employed for mid-infrared applications, it remains a daunting challenge to search for other materials that possess suitable bandgaps in this wavelength range. Here, we demonstrate experimentally for the first time that twodimensional (2D) atomically thin $\mathrm{PtSe}_{2}$ has a variable bandgap in the mid-infrared via layer and defect engineering. Here, we show that bilayer $\mathrm{PtSe}_{2}$ combined with defects modulation possesses strong light absorption in the mid-infrared region, and we realize a mid-infrared photoconductive detector operating in a broadband mid-infrared range. Our results pave the way for atomically thin 2D noble metal dichalcogenides to be employed in high-performance mid-infrared optoelectronic devices.

\footnotetext{
${ }^{1}$ Centre for OptoElectronics and Biophotonics, School of Electrical and Electronic Engineering \& The Photonics Institute, Nanyang Technological University, 50 Nanyang Avenue, Singapore 639798, Singapore. ${ }^{2}$ Centre for Programmable Materials, School of Materials Science and Engineering, Nanyang Technological University, 50 Nanyang Avenue, Singapore 637371, Singapore. ${ }^{3}$ Centre for Advanced 2D Materials and Graphene Research Centre, National University of Singapore, Singapore 117546, Singapore. ${ }^{4}$ Department of Physics, National University of Singapore, Singapore 117542 , Singapore. ${ }^{5}$ School of Physics Science, CAS Key Laboratory of Vacuum Physics, University of Chinese Academy of Sciences, 100049 Beijing, China. ${ }^{6}$ National Institute of Advanced Industrial Science and Technology (AIST), Tsukuba 305-8565, Japan. ${ }^{7}$ NOVITAS, Nanoelectronics Centre of Excellence, School of Electrical and Electronic Engineering, Nanyang Technological University, Singapore 639798, Singapore. These authors contributed equally: Xuechao Yu, Peng Yu. Correspondence and requests for materials should be addressed to Z.L. (email: z.liu@ntu.edu.sg) or to Q.J.W. (email: qjwang@ntu.edu.sg)
} 
T he enthusiasm for research on mid-infrared (mid-IR) radiation that contains fingerprints of the molecular vibrations and transparent windows in the atmosphere is driven by the tremendous applications ranging from optical communications, infrared imaging to analytical sciences ${ }^{1}$. Commercial Mercury Cadmium Telluride (MCT) made of CdTe and HgTe alloy, by far the paradigmatic candidate for mid-IR applications, exhibits robust performances, and the bandgap is varied by adjusting the alloy compositions, or by externally changing the operation temperatures ${ }^{2}$. InSb is also an extensively explored narrow bandgap semiconductor for short-wave mid-IR applications, and the operation regime can be extended to $\sim 7 \mu \mathrm{m}$ via nitrogen doping ${ }^{3}$. Apart from these materials based on interband transitions, compound semiconductor superlattices with alternate layers of different semiconductors have been developed in the past few decades for mid-IR optoelectronics, based on intersubband transitions ${ }^{4,5}$. The present mid-IR materials are intensively stuck with environmental toxicity, high cost, and complex fabrication processes.

The urgency for exploring alternative candidate materials in the mid-IR range has been raised with insistence. The twodimensional (2D) material is an emerging platform with atomical thickness and exceptional properties, which has revolutionized material science, chemistry, and physics ${ }^{6}$. For instance, graphene, a $2 \mathrm{D}$ crystal with honeycomb-arranged carbon atom layer, has attracted enormous interests in the mid-IR optoelectronics due to its unique optoelectronic properties, such as broadband absorption, ultrahigh carrier mobility, etc. ${ }^{7}$. However, the lowabsorption coefficient and short-carrier lifetime are major constraints for developing versatile mid-infrared optoelectronic devices, even though graphene nanoribbons have been employed to either open a suitable bandgap or excite graphene plasmons for enhancing the mid-infrared light-matter interactions $s^{8-10}$. The existing challenges stimulate the search for alternative $2 \mathrm{D}$ materials with an intrinsic narrow bandgap. Black phosphorene (BP), which is recently rediscovered from the perspective of a $2 \mathrm{D}$ layered material, has a layer-dependent bandgap from 0.3 to 2.0 $\mathrm{eV}$. Although multilayer BP is suitable for mid-infrared photodetectors and modulators ${ }^{11-13}$, it only covers up to a wavelength of $\sim 4.1 \mu \mathrm{m}(h v \sim 0.3 \mathrm{eV})$ and suffers from relatively poor environmental stability $^{12}$. On the other hand, traditional $2 H$-phase transition metal dichalcogenides (TMDCs), $\mathrm{MX}_{2}(\mathrm{M}=\mathrm{Mo}, \mathrm{W}$; $\mathrm{X}=\mathrm{S}, \mathrm{Se}$ ), are not suitable for mid-infrared photonic applications because of the wide bandgap that only covers the visible range ${ }^{14}$. As a result, most of the currently explored TMDCs are not appropriate for this lower-energy spectral range via interband optical transitions.

Extending 2D materials to the spectral range of mid-infrared irradiation has experienced an impetus in terms of developing novel theoretical and technological strategies to provide new insights into related phenomena ${ }^{15}$. Recently, the noble metal dichalcogenides (such as $\mathrm{PtS}_{2}, \mathrm{PtSe}_{2}, \mathrm{PdS}_{2}, \mathrm{PdSe}_{2}$, etc.) present a foray into promising candidates due to their potentially suitable bandgaps for the mid-infrared photonic and optoelectronic applications, and high environmental stability ${ }^{16}$. In this work, we successfully demonstrate high-performance mid-infrared materials from bilayer $\mathrm{PtSe}_{2}$ mechanically exfoliated from its bulk counterpart, which is synthesized by a self-designed chemical vapor transport (CVT) method. Compared with other fabrication strategies that we have also demonstrated in this work, such as chemical vapor deposition (CVD) and direct selenization of platinum substrate, our designed CVT method has the most leverage to realize high-quality, large-area, and layer-controlled $\mathrm{PtSe}_{2}$ atomic layers for optoelectronic devices. Intriguingly, the bandgap can also be efficiently tuned through defect engineering via growth-condition controls and/or plasma treatments, which is verified by both theoretical calculations and mid-IR optoelectronic measurements. High-performance photoconductive detectors with a remarkable photoresponse in a broadband spectrum region from the visible to the mid-infrared are achieved, using bilayer $\mathrm{PtSe}_{2}$ as an activating material. Our results reveal a roadmap toward future development of high-performance $2 \mathrm{D}$ material for photonic devices, such as mid-infrared cameras, photodetectors, modulators etc.

\section{Results}

PtSe2 atomic layers fabrication methods and characterizations. $\mathrm{PtSe}_{2}$ crystal possesses a typical $1 T$-type hexagonal crystal structure with $P-3 m 1$ space group, as shown in Fig. 1a, b. It consists of three atomic layers stacking in the order of Se-Pt-Se, which are held together by weak Van Der Waals forces. Within a single $\mathrm{PtSe}_{2}$ layer, Se atoms are strongly bonded with $\mathrm{Pt}$ atoms forming an octahedral prismatic local coordination structure, where $\mathrm{Pt}$ atoms lie at the center of the coordination unit. The $\mathrm{PtSe}_{2}$ octahedra are connected to each other by sharing Se-Se edges, as shown in Supplementary Fig. 1. The layered crystal structure of $\mathrm{PtSe}_{2}$, analogous to the traditional TMDCs, enables the fabrication of atomic layers by proper exfoliation strategies. However, obtaining high-quality $\mathrm{PtSe}_{2}$ atomic layers with designed bandgap and thickness is critical toward its practical applications. In this paper, we report a custom-designed CVT setup to synthesize $\mathrm{PtSe}_{2}$ single crystal, followed by a mechanical exfoliation method to fabricate the atomic layers, as shown in Fig. 1c. The source material evaporation and reaction process are controlled by adjusting the temperature zone in the vacuum tube. Highresolution transmission electron microscope (HRTEM) of $\mathrm{PtSe}_{2}$ atomic layers indicates the high-quality of the fabricated thin layers. For comparison, we also design two alternative methods to synthesize $\mathrm{PtSe}_{2}$ atomic layers: CVD and direct selenization of $\mathrm{Pt}$ substrate. In the CVD process, $\mathrm{PtSe}_{2}$ atomic layers are achieved by evaporating $\mathrm{PtCl}_{2}$ and selenide source materials, followed by reaction and deposition on the $\mathrm{Si} / \mathrm{SiO}_{2}$ wafer. The Raman spectrum, as shown in Fig. 1d, indicates sharp characteristic peaks; however, the HRTEM image shows some large holes and structural defects. In addition, direct selenization of platinum substrate, as shown in Fig. 1e, is also proved to be an efficient strategy to synthesize high-quality $\mathrm{PtSe}_{2}$ thin films, in which the thickness can be tuned by the growth temperature. The Raman spectrum shows similar $E_{\mathrm{g}}$ and $A_{1 \mathrm{~g}}$ peaks, and the HRTEM image is found to be fully consistent with the crystal structure of $1 T-\mathrm{PtSe}_{2}{ }^{17}$.

From the above three methods and the corresponding characterizations presented here, we can state that the CVD method is feasible to fabricate $\mathrm{PtSe}_{2}$ atomic layers from monolayer to few layers on the conventional $\mathrm{Si} / \mathrm{SiO}_{2}$ wafer, but the size and quality of the $\mathrm{PtSe}_{2}$ atomic layers are not suitable for optoelectronic device applications. Even though the direct selenization of $\mathrm{Pt}$ substrate results in large size and high-quality $\mathrm{PtSe}_{2}$, only thin films of $\mathrm{PtSe}_{2}$ on the Pt substrate can be fabricated by this method, which is also not acceptable for optoelectronic device applications. On the contrary, the CVT method followed by a flexible mechanical exfoliation process is capable of fabricating $\mathrm{PtSe}_{2}$ atomic layers from monolayer to few layers with high-quality. As a result, we employ the designed CVT method, as shown in Fig. 1c., to explore the mid-IR optoelectronics properties in this work.

The as-grown single crystals of $\mathrm{PtSe}_{2}$, up to a size of $2-3 \mathrm{~mm}$, is shown in Fig. 2a with a flat and wrinkle-free surface. The energydispersive X-ray spectroscopy (EDS) spectrum in Fig. $2 \mathrm{~b}$ indicates the pure chemical composition of the exfoliated $\mathrm{PtSe}_{2}$ flakes without the residues of the catalyzers. However, the atomic ratio is smaller than that in the perfect $\mathrm{PtSe}_{2}$ crystal, indicating that $\mathrm{Se}$ 
is normally deficient in the crystal fabricated by the CVT method. Figure $2 \mathrm{c}$ shows the X-ray diffraction (XRD) pattern of the fabricated $\mathrm{PtSe}_{2}$ crystals. The X-ray pattern matches very well with the previous studies ${ }^{17,18}$, and the three major characteristic peaks are observed, while other peaks are counteracted due to the layered structure and special orientation (001) of the $\mathrm{PtSe}_{2}$ crystal. In addition, the strong, narrow peaks for (001), (012), and (111) demonstrate the high crystal quality of the synthesized samples. The EDS further confirms that pure phase of as-grown $\mathrm{PtSe}_{2}$ has been successfully obtained by the CVT method. The quality of $\mathrm{PtSe}_{2}$ sample is systematically characterized by TEM, as shown in Fig. 2d, to clarify its quality and atomic structure, from which we can obtain the lattice constant of $\sim 3.8 \AA$, in excellent agreement with the theoretical value and previous reports ${ }^{17}$. The TEM, EDS, as well as the XRD pattern in Fig. 2 demonstrates the successful growth of highly crystallined $\mathrm{PtSe}_{2}$ flakes.
$\mathrm{PtSe}_{2}$ atomic layers are mechanically exfoliated from the bulk crystals by the Scotch tape method, and transferred onto a silicon wafer with a 285-nm thermal oxidation layer. The optical microscope images of the exploited monolayer, bilayer, and trilayer $\mathrm{PtSe}_{2}$ on the $\mathrm{Si} / \mathrm{SiO}_{2}$ wafer are shown in Fig. 3a-c. The thickness of the samples can be determined by the AFM height profiles, measured from the red lines crossing the flakes. The thickness of the monolayer $\mathrm{PtSe}_{2}$ is about $0.63 \mathrm{~nm}$, as shown in Fig. 3a, where a slight dip in the boundaries is caused by the absorbed molecules and/or residues. As shown in Fig. 3b, c, the thicknesses of the bilayer and the trilayer $\mathrm{PtSe}_{2}$ are measured to be about $1.04 \mathrm{~nm}$ and $1.62 \mathrm{~nm}$, respectively, which almost increase linearly from monolayer to trilayer. The layer-dependent properties can also be characterized by Raman spectroscopy, similar to other $2 \mathrm{D}$ material characterizations ${ }^{18}$. The synthesized bulk $\mathrm{PtSe}_{2}$ and atomic layers show two main Raman peaks near a

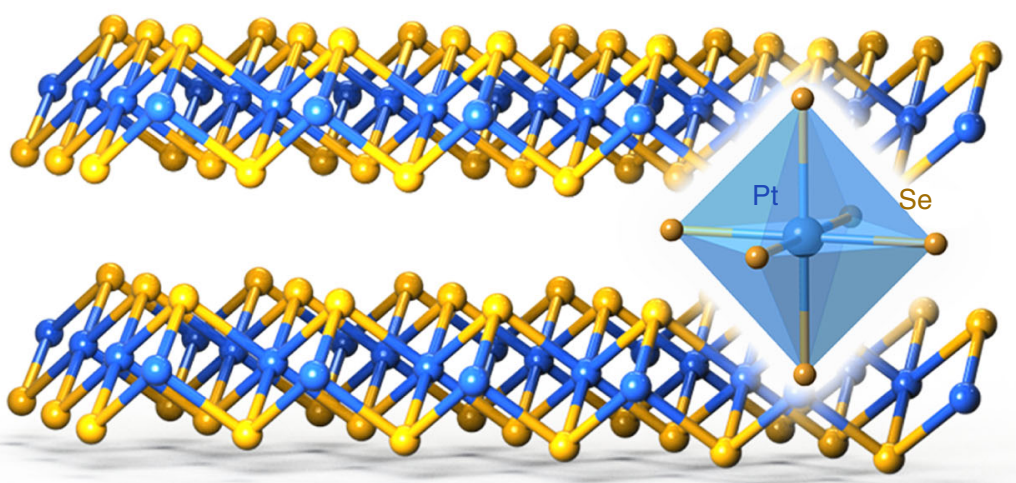

b

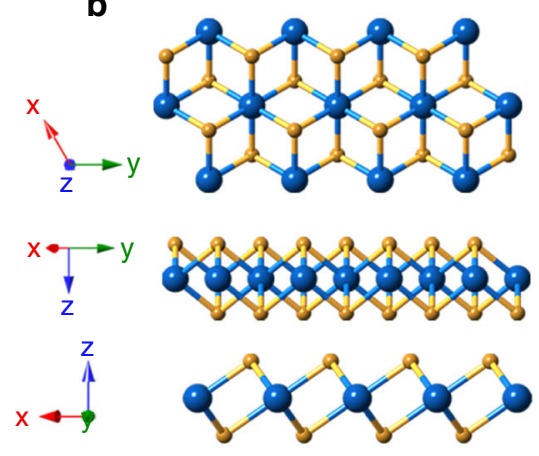

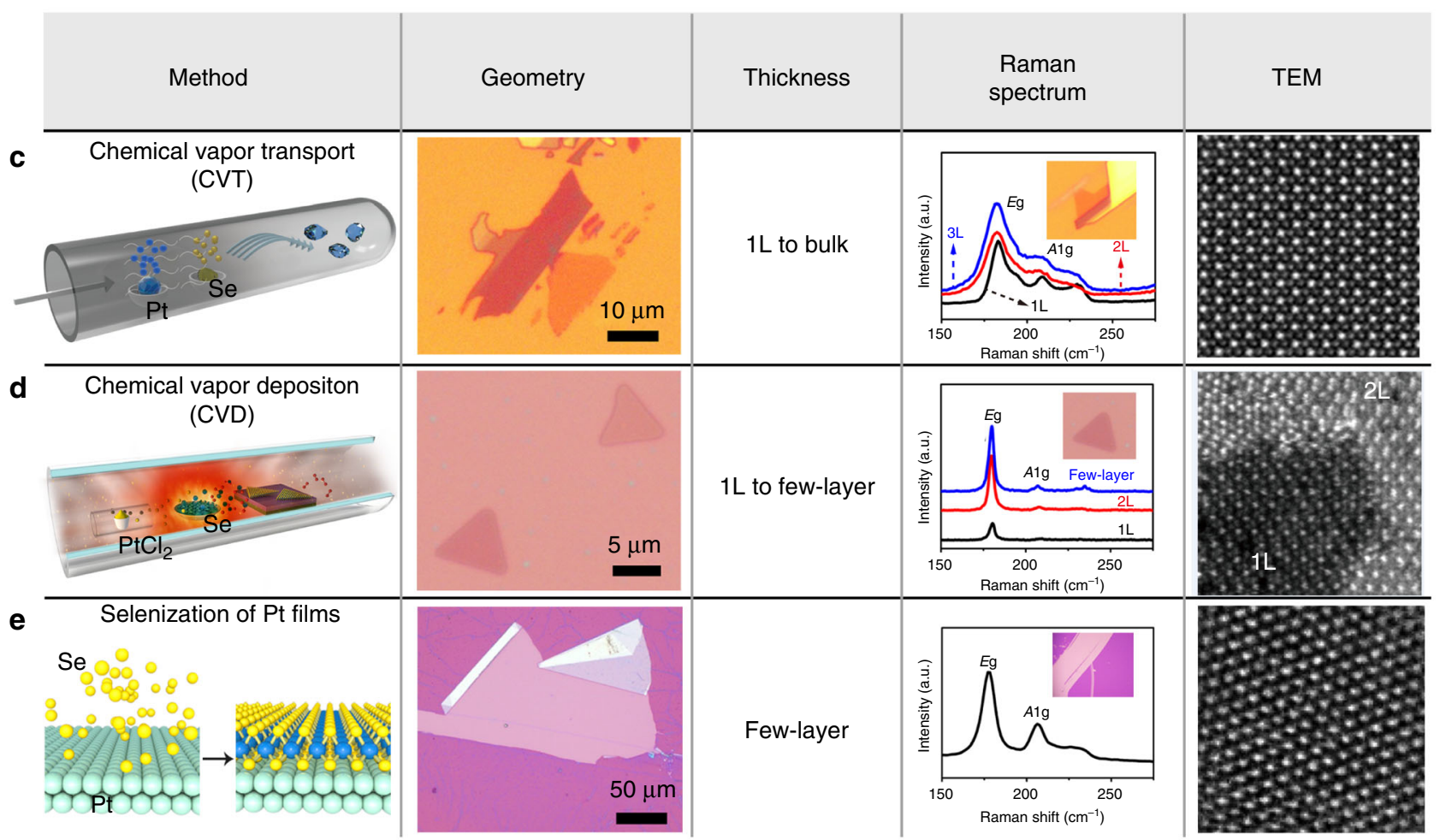

Fig. 1 Synthesis and characterizations of $\mathrm{PtSe}_{2}$ by three different fabrication methods. a Crystal structure of $\mathrm{PtSe} \mathrm{e}_{2}$ and $\mathbf{b}$ its three-dimensional (3D) side views. c Illustrative diagram of experimental setup for the growth of $\mathrm{PtSe} \mathrm{e}_{2}$ single crystals by CVT method, optical microscope image, Raman spectroscopy, and HRTEM image of atomic PtSe $e_{2}$ layers via mechanical exfoliation. d Schematic diagram of experimental setup, optical microscope image, Raman spectroscopy, and HRTEM image of atomic PtSe $e_{2}$ layers fabricated by CVD method. e Growth process, optical microscope image, Raman spectroscopy, and HRTEM image of the few-layer (FL) $\mathrm{PtSe}_{2}$ via direct selenization of platinum substrate 

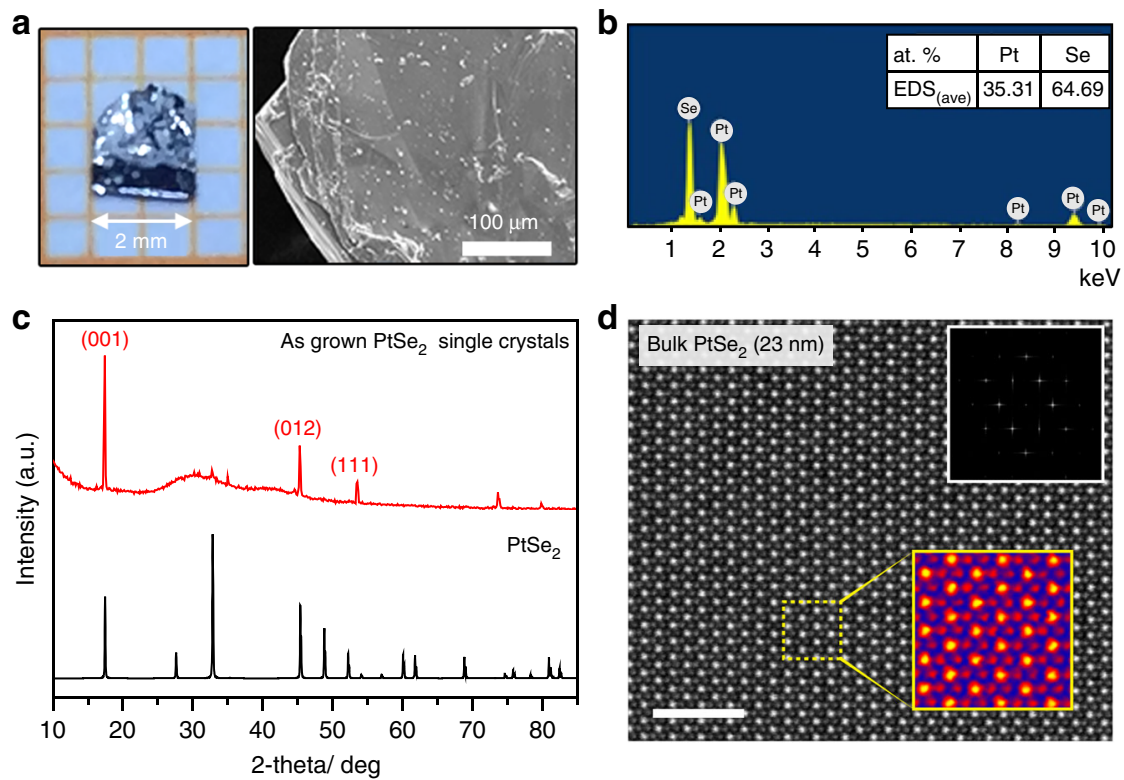

Fig. 2 Synthesis and characterization of $\mathrm{PtSe}_{2}$ crystals fabricated through the CVT method. a Optical microscope image and scanning electron microscope (SEM) image of the synthesized PtSe $e_{2}$ flakes. $\mathbf{b}$ EDS of the $\mathrm{PtSe}_{2}$ flakes indicating the atomic ratio of Pt/Se. $\mathbf{c}$ X-ray diffraction spectra of the CVT grown, large $\mathrm{PtSe}_{2}$ crystals (up, red), and $1 T-\mathrm{PtSe}_{2}$ standard card (bottom, black). d Transmission electron microscopy characterization, Fast Fourier transform of the atomic resolution scanning transmission electron microscope (STEM), Z-contrast image (the upper right corner), and STEM Z-contrast image of the exfoliated $\mathrm{PtSe}_{2}$ few layers. The scale bar is $2 \mathrm{~nm}$

$200 \mathrm{~cm}^{-1}$ and $300 \mathrm{~cm}^{-1}$, as shown in Fig. 1c and Supplementary Figs. 3-4, which were defined as $E_{\mathrm{g}}$ mode and $A_{\mathrm{lg}}$ mode vibration, respectively ${ }^{17}$. On the other hand, the interlayer shear modes of atomic-layered $\mathrm{PtSe}_{2}$ in the ultra-low-frequency Raman spectrum, as shown in Supplementary Fig. 5, clearly indicate the layered structure of $\mathrm{PtSe}_{2}$ flakes. And it could be used to accurately determine the layer number of the few-layer $\mathrm{PtSe}_{2}$, as shown in Supplementary Fig. 6. In addition, it is also clear, as shown in the HRTEM image in Fig. 3d, that there are a considerable number of Se vacancies randomly distributed in the crystal lattice. These defects play an important role in the bandgap tuning and light absorption in the mid-IR regime of the $\mathrm{PtSe}_{2}$ atomic layers, and will be discussed in detail later.

To understand the layer-dependent band structure and bandgap evaluation of $\mathrm{PtSe}_{2}$, first-principles calculations are performed. It is shown that the monolayer and bilayer $\mathrm{PtSe}_{2}$ are semiconductors with indirect bandgap of $\sim 1.2$ and $\sim 0.3 \mathrm{eV}$, respectively (see Fig. $3 \mathrm{e}-\mathrm{g}$ ), which are consistent with the earlier reports ${ }^{16,19}$. It becomes metallic without a bandgap for thicker $\mathrm{PtSe}_{2}$ layers. The slight mismatch in our calculations might be caused by the scanning resolution limit of the Vienna Ab Initio Simulation Package projector for such a narrow bandgap semiconductor, as shown in Supplementary Figs. 1 and 2. The narrow bandgap of the bilayer $\mathrm{PtSe}_{2}$ makes it suitable for light absorption in the mid-infrared $(\lambda>4 \mu \mathrm{m})$ region, filling up the gap between graphene and commonly explored BP and TMDCs. Thus, bilayer $\mathrm{PtSe}_{2}$ is an appropriate $2 \mathrm{D}$ semiconducting candidate with interband transition for mid-infrared photonic and optoelectronic applications.

Optoelectronic properties of monolayer and bilayer PtSe2 FET. To demonstrate the optoelectronic properties of the atomically thin $\mathrm{PtSe}_{2}$ layers, we investigated mid-infrared photodetection with $\mathrm{PtSe}_{2}$ FET as a demonstrative example to show its practical applications. The electrical contacts made of $\mathrm{Ti} / \mathrm{Au}(20 \mathrm{~nm} / 80$ $\mathrm{nm}$ ) layers are deposited by the e-beam evaporation, after standard photolithography procedures. Heavily doped Si substrate is used as the back gate. The $I_{\mathrm{D}}-V_{\mathrm{D}}$ and $I_{\mathrm{D}}-V_{\mathrm{G}}$ curves are measured by the Agilent probe station, as shown in Supplementary Figs. 9-11. All the measurements are conducted in the dark under room temperature. One striking observation is the layer-dependent metallic to semiconducting phase transition: trilayer $\mathrm{PtSe}_{2}$ was metallic, while bilayer and monolayer $\mathrm{PtSe}_{2}$ were semiconducting, which is consistent with the theoretical predictions and experimental results ${ }^{17,19}$. It also depicts that both bilayer and monolayer $\mathrm{PtSe}_{2}$ exhibit classical n-type semiconducting behaviors. Additionally, the carrier mobilities of bilayer and monolayer $\mathrm{PtSe}_{2}$ can be calculated to be 8.6 and 1.7 $\mathrm{cm}^{2} \mathrm{~V}^{-1} \mathrm{~s}^{-1}$, respectively, which are on par with previous reports about $\operatorname{InSe} e^{20,21}, \operatorname{In}_{2} \mathrm{Se}_{3}{ }^{22,23}$, and so on. The decrease of the mobility from bilayer $\mathrm{PtSe}_{2}$ FET to monolayer $\mathrm{PtSe}_{2}$ FET might be attributed to the surrounding conditions or the charge transfer from neighboring adsorbates and the substrates ${ }^{24}$.

We then discuss the photodetection performance of monolayer $\mathrm{PtSe}_{2}$ FET. The measurement setup is shown in Supplementary Note 4 , and the power concentration of the laser illumination is kept in constant for time-resolved measurement, as shown in Fig. 4a, b. The time-resolved photoresponse-measurement results of monolayer $\mathrm{PtSe}_{2}$ are shown in Fig. 4a,b and Supplementary Table 1 . The photocurrent is defined as $I_{\mathrm{ph}}=I_{\text {illum }}-I_{\text {dark }}$, where $I_{\text {dark }}$ is the dark current and measured to be $28.5 \mathrm{nA}$ and $5.5 \mathrm{nA}$ for $632 \mathrm{~nm}$ and $1.47 \mu \mathrm{m}$ illumination, respectively. The photodetectors based on monolayer $\mathrm{PtSe}_{2}$ show photoresponses of 0.9 and $0.15 \mathrm{~A} \mathrm{~W}^{-1}$ for $632 \mathrm{~nm}$ and $1.47 \mu \mathrm{m}$ illumination, respectively. However, negligible photoresponse under a mid-infrared laser illumination $(\lambda=10 \mu \mathrm{m})$ is observed because the bandgap of monolayer $\mathrm{PtSe}_{2}\left(E_{\mathrm{g}} \sim 1.2 \mathrm{eV}\right)$ far exceeds the photon energy of the incident laser.

On the other hand, bilayer $\mathrm{PtSe}_{2}$ FET with controlled defect engineering exhibits a much higher photoresponse, compared with the monolayer $\mathrm{PtSe}_{2} \mathrm{FET}$, as shown in Fig. 4d, e. For instance, the photoresponsivities $\left(R_{\text {res }}\right)$ in the visible and near- 

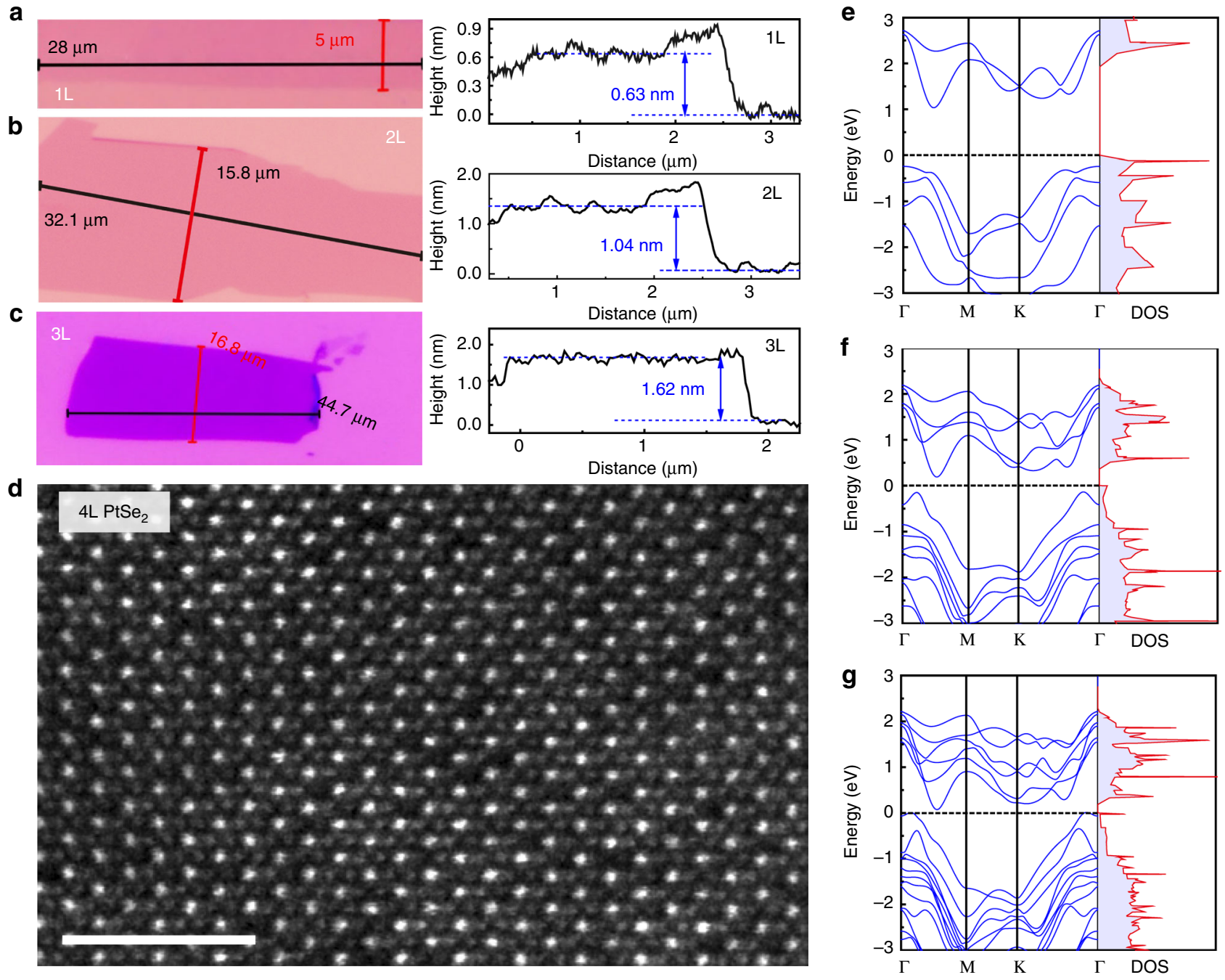

Fig. 3 Characterization of atomically thin $\mathrm{PtSe}_{2}$ and the bandgap evolution obtained by first-principles calculations. a-c Optical microscope images and atomic force microscope (AFM) height profiles of monolayer, bilayer, and trilayer PtSe 2 . $\mathbf{d}$ HRTEM image of the atomic layered of PtSe $e_{2}$. The scale bar is 2 $\mathrm{nm}$. e-g Density-of-states (DOS) of defect-free monolayer (e), bilayer (f), and trilayer $\mathrm{PtSe}_{2}$ (g) by first-principles calculations

infrared regions are about $6.25 \mathrm{~A} \mathrm{~W}^{-1}$ and $5.5 \mathrm{~A} \mathrm{~W}^{-1}$, respectively. Importantly, we also observe a strong photoresponse in the mid-infrared region $(\lambda=10 \mu \mathrm{m})$, as shown in Fig. $4 \mathrm{~d}$, and a photoresponsivity of $\sim 4.5 \mathrm{~A} \mathrm{~W}^{-1}$ as shown in Supplementary Table 2, which corresponds to an internal gain of 17.5 (see supplementary materials). The photoresponse of the bilayer $\mathrm{PtSe}_{2}$ is about three orders higher than common graphene photodetectors and on par with the commercial MCT and QWIP detectors. Furthermore, the rise time and decay time can be fitted by the following equation ${ }^{25}: I_{\text {rise }}=I_{0}-A \exp \left(-\left(t-t_{1}\right) / \tau_{1}\right)$ and $I_{\text {decay }}=I_{0}+B \exp \left(-\left(t-t_{2}\right) / \tau_{2}\right)$, where $\tau$ is the time constant and $t$ is the time when the laser is switched on or off. $A$ is the scaling constant. The fitted characteristic photoresponse time coefficients, $\tau_{1}$ and $\tau_{2}$ are $1.2 \pm 0.1 \mathrm{~ms}$ for rise time and $1.2 \pm 0.1 \mathrm{~ms}$ for fall time, respectively, which is better than those of other types of 2D semiconducting photodetectors, such as the reported $\mathrm{MoS}_{2}$ photodetectors ${ }^{24,26,27}$ and BP photodetectors ${ }^{28-32}$. Besides, the rise time and fall time can also be calculated by the time period between $10 \%$ and $90 \%$ of the current in the rising and falling curves, as shown in Fig. $4 \mathrm{~b}$, e. The rise time $\left(\tau_{1}=1.1 \mathrm{~ms}\right)$ and fall time $\left(\tau_{2}=1.2 \mathrm{~ms}\right)$ are consistent with the former-fitted values. Last but not least, it is important to mention that the detectivity $\left(D^{*}\right)$ of $\mathrm{PtSe}_{2}$-based mid-infrared photodetector, which is an essential figure of merit for the detector, is limited by the $1 / f$ noise other than the shot noise in our operation conditions. We measure the $1 / f$ noise of the device in the frequency range from 1 $\mathrm{Hz}$ to $10 \mathrm{kHz}$, as shown in Supplementary Fig. 12. It is clearly indicated that the low-frequency $1 / f$ noise prevails in our photodetectors, similar to other $2 \mathrm{D}$ systems ${ }^{33}$. The detectivity can be calculated based on the current noise spectrum, as shown in Supplementary Fig. 13, and noise the equivalent power (NEP, supplementary materials) in the current noise spectra by $D=\sqrt{A B} / N E P$, where $A$ is the area of the detector and $B$ is the bandwidth. The detectivity is calculated to be $\sim 7 \times 10^{8}$ Jones $\left(\mathrm{cm} \mathrm{Hz}^{1 / 2} \mathrm{~W}^{-1}\right)$ under $10 \mu \mathrm{m}$ quantum cascade laser (QCL) illumination, which is at the same level as that of commercial mid-infrared photodetector, in this wavelength range ${ }^{2}$.

In addition, the power-dependent photocurrent can be expressed by the power law, $I_{\mathrm{PC}}=C P^{\gamma}$ ( $C$ is a constant and $P$ is the illumination power), as shown in Fig. $4 \mathrm{c}$, f. For the laser at the operating wavelengths of $632 \mathrm{~nm}$ and $1.47 \mu \mathrm{m}, \gamma$ is 0.92 and 0.7 for monolayer $\mathrm{PtSe}_{2} \mathrm{FET}$, respectively, indicating that the recombination kinetics of the photocarriers involves both traps/ defects states and photogenerated carrier interactions ${ }^{34}$. In comparison, the value of $\gamma$ is $1.07,0.96$, and 0.93 for $632 \mathrm{~nm}$, $1.47 \mu \mathrm{m}$, and $10 \mu \mathrm{m}$ for bilayer $\mathrm{PtSe}_{2} \mathrm{FET}$, respectively. The relatively higher value for bilayer $\mathrm{PtSe}_{2}$ FET might be ascribed to mid-gap states and substrate effects, compared to the monolayer 

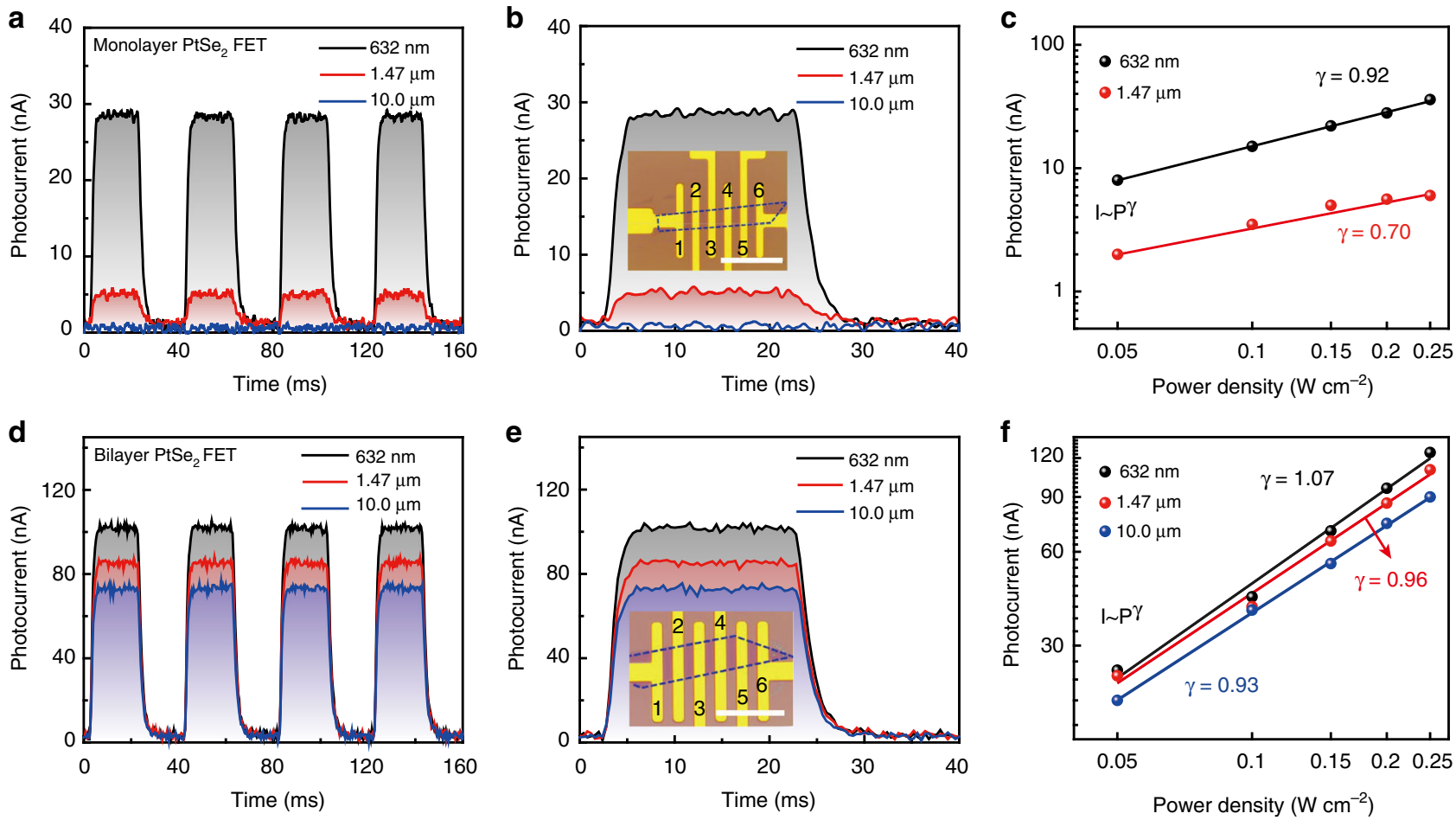

Fig. 4 Optoelectronic properties of the monolayer and the bilayer $\mathrm{PtSe}_{2}, \mathbf{a}, \mathbf{b}$ Time-resolved photoresponse of the monolayer PtSe $\mathrm{e}_{2}$ field effect transistor (FET) devices for a bias voltage of $0.1 \mathrm{~V}$ and zero gate voltage under laser illumination with a wavelength of $632 \mathrm{~nm}, 1.47 \mu \mathrm{m}$ and $10 \mu \mathrm{m}$, respectively. c Power dependence of the monolayer PtSe $\mathrm{F}_{2}$ FET devices under laser illumination recorded at $V_{D}=0.1 \mathrm{~V}$. $\mathbf{d}$, e Time-resolved photoresponse of the bilayer PtSe2 FET device. The laser illuminations in $\mathbf{a}, \mathbf{b}, \mathbf{d}$ and $\mathbf{e}$ are kept in a constant of $0.2 \mathrm{~W} \mathrm{~cm}^{-2}$ for three different lasers. $\mathbf{f}$ Power dependence of the bilayer $\mathrm{PtSe}_{2}$ FET devices under laser illumination recorded at $V_{D}=0.1 \mathrm{~V}$. The insert figures in $\mathbf{b}$ and $\mathbf{e}$ are the microscopic image of the monolayer and bilayer $\mathrm{PtSe}_{2} \mathrm{FET}$, respectively, measured in the experiment. The blue envelope area is the $\mathrm{PtSe}_{2}$ sample. The scale bar is $20 \mu \mathrm{m}$

devices $^{24}$. Further investigation is needed to interpret the complex-carrier recombination and scattering processes of the photogenerated electron/hole pairs. Furthermore, the increase of the photocurrent with the incident laser power, as shown in Fig. 4c, f, together with the laser wavelength-dependent photoresponse, as shown in Supplementary Fig. 14, indicates increase in the number of photogenerated carriers with an increase in the incident laser power ${ }^{26}$.

It is also noted that, the response speed of the bilayer $\mathrm{PtSe}_{2}$ FET is quite fast at the millisecond level, which surpasses the previously reported results of graphene nanostructured photodetectors $^{35,36}$ and 2D semiconducting photodetectors ${ }^{26}$. In addition, the mid-infrared photodetection performance can be further improved by dielectric engineering and surface engineering strategies ${ }^{37}$. For example, the mobility of the $\mathrm{PtSe}_{2}$ FET could be enhanced in the high-dielectric environment due to the reduction of Coulomb interactions among the carriers in the channel. Furthermore, the slow response speed might be originated from the trapping states in the active channel, which can be optimized by surface-engineering methods, such as chemical treatment and trapping molecular/film decorations ${ }^{36,37}$.

\section{Discussion}

As shown in Fig. 5a, mid-infrared detection with 2D materials has been a challenging task due to the lack of suitable candidates, besides graphene sheet and graphene nanostructures ${ }^{35}$. In this work, we report bilayer $\mathrm{PtSe}_{2}$ with designed defect engineering, for the first time, as a broadband mid-infrared photodetector operated at room temperature, as shown in Fig. 5b and Supplementary Table 2. The responsivity and the response speed also far exceeds those obtained in the recently discovered black arsenic phosphorus in the $3-5 \mu \mathrm{m}$ range ${ }^{38}$. The broadband photoresponse of bilayer $\mathrm{PtSe}_{2}$ FET is originated from the defectinduced bandgap decrease. We now turn to the discussion of the additional characterizations and the reasons that address potential explanations of these findings. The quality and stoichiometric ratio between Pt to Se are further monitored by XPS, as shown in Fig. 5c, d and Supplementary Fig. 7. From the calculations, and based on the XPS spectra, we obtained the atomic ratio of Se/ Pt of 64/36, which agrees well with the EDS spectrum and HRTEM images, as shown in Fig. 2 (see supplementary materials). In order to examine the role of defects in the electronic properties of bilayer $\mathrm{PtSe}_{2}$, we developed a technique to control the defect concentration via argon (Ar) plasma treatment in this home-made system.

After Ar plasma treatment with controlled dosages, the defect concentration and evolution of the bandgap of bilayer $\mathrm{PtSe}_{2}$ with the increase of defect concentration are characterized by the XPS spectra and FTIR absorption measurements, as shown in Supplementary Fig. 8, where the absorption cut-off wavelength dramatically shifts from $1210 \mathrm{~cm}^{-1}$ to $870 \mathrm{~cm}^{-1}$ (corresponding to photon energy from $150 \mathrm{meV}$ to $110 \mathrm{meV}$ ) with the defect concentration increase from $3 \%$ to $7.9 \%$. Consequently, the bandgap evolution with introducing Se-vacancy through controlled $\mathrm{Ar}$ plasma treatment is also verified by the photoresponses $(10 \mu \mathrm{m}$ laser illumination) of the bilayer $\mathrm{PtSe}_{2}$ samples, as shown in Supplementary Fig. 15, where the photoresponse increases dynamically with the increase in the defect concentration. Furthermore, we investigate the bandgap evolution of bilayer $\mathrm{PtSe}_{2}$ with different concentrations of Se vacancies, using firstprinciples calculations; the results are shown in Fig. 5e, f. The decrease of the bandgap of bilayer $\mathrm{PtSe}_{2}$ with the increase of the defect concentration can be explained as below. The valence band maximum in bilayer $\mathrm{PtSe}_{2}$ is mainly contributed by $5 d$ orbitals of 

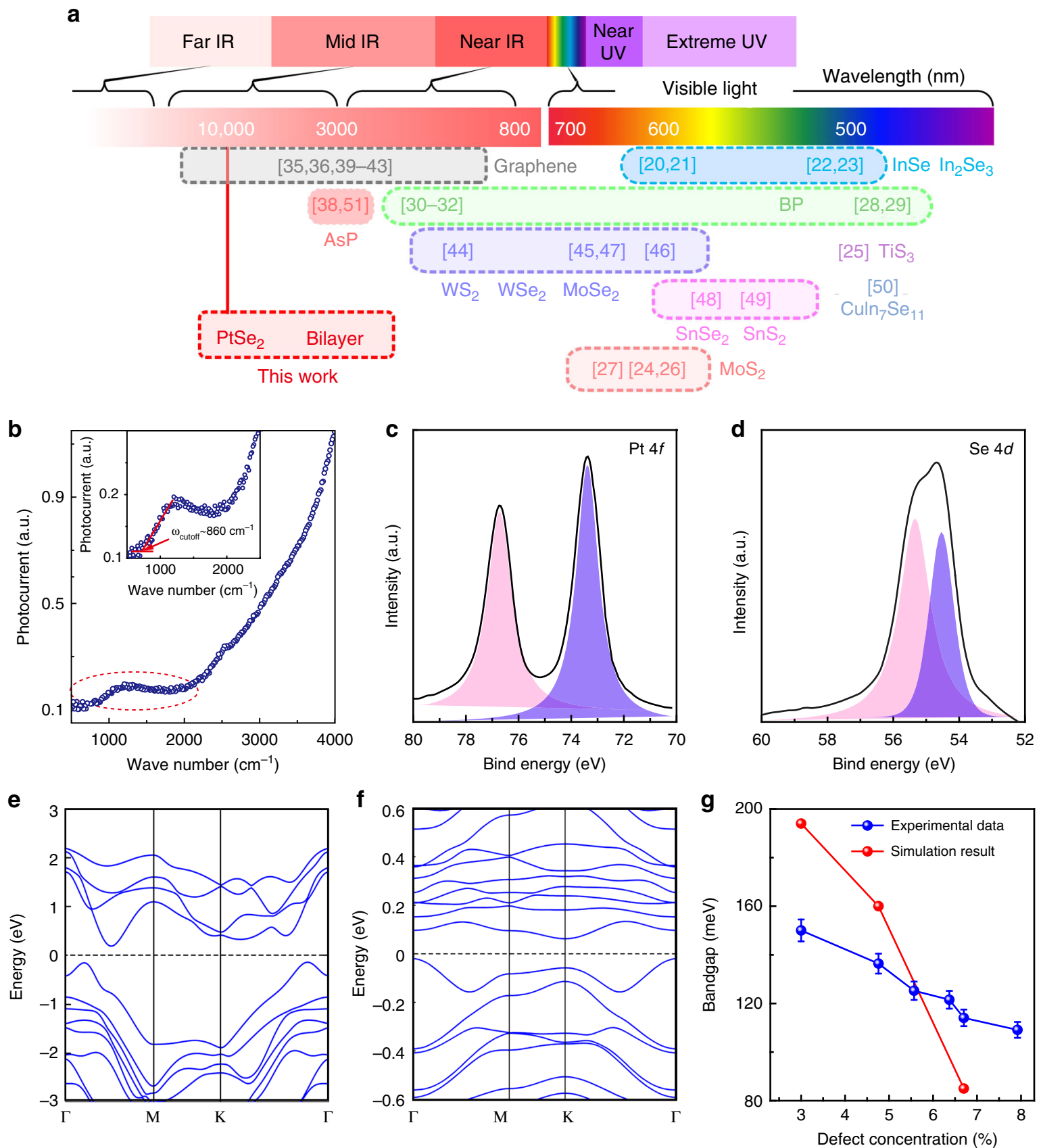

Fig. 5 Narrow bandgap bilayer $\mathrm{PtSe}_{2}$ via defect engineering. a Comparison of photodetectors operated at different wavelengths, based on two-dimensional materials, such as graphene $35,36,39-43, \mathrm{MoS}_{2}{ }^{24,26,27}$, black phosphorene ${ }^{28-32}, \mathrm{WS}_{2}{ }^{44,45}, \mathrm{WSe}_{2}{ }^{46}, \mathrm{MoSe}_{2}{ }^{47}, \mathrm{SnSe}_{2}{ }^{48}, \mathrm{SnS}_{2}{ }^{49}, \operatorname{InSe}^{20,21}, \mathrm{In}_{2} \mathrm{Se}_{3} 22,23, \mathrm{TiS}_{3}{ }^{25}$, $\mathrm{Culn}_{7} \mathrm{Se}_{11} 50$, and black AsP 38,51 . The mid-infrared range is rarely explored besides graphene. b Photoresponse spectrum of the photodetector based on the Ar plasma treatment bilayer PtSe 2 FET (20 s), measured by FTIR internal source. c, d X-ray photoelectron spectroscopy (XPS) spectrum of Pt $4 f(\mathbf{c})$ and Se $3 d$ (d) core level peaks of $\mathrm{PtSe}_{2}$ with Se vacancies. e, $\mathbf{f}$ Density of states (DOS) of bilayer $\mathrm{PtSe}_{2}$ with $3 \%$ (e) and $6.8 \%$ (f) Se vacancy concentrations by first-principles calculations. $\mathbf{g}$ The experimental (blue) and theoretical (red) bandgap evolution of bilayer $\mathrm{PtSe}_{2}$ with different Se vacancy concentrations

Pt atoms that contains rich $d$-electrons. As a result, the interaction of the $d$ band of $\mathrm{Pt}$ atom and the $p_{z}$ band of Se atom thermodynamically favors to form $s p^{3} d^{2}$ hybridization ( $1 T$ phase), in which, less $d$ orbitals are involved and the bond between Pt and Se are weaker than those of group- 5 or group- 6 TMDCs, such as $\mathrm{MoS}_{2}, \mathrm{WS}_{2}$ with $s p d^{4}$ hybridization in $2 \mathrm{H}$ phase. During the synthesis procedure and Ar plasma treatment, high temperature is required to exceed the melting temperature of $\mathrm{Pt}$, which breaks the $\mathrm{Pt}-\mathrm{Se}$ bond and causes Se vacancies. From the DOS of bilayer $\mathrm{PtSe}_{2}$ with defects, it is shown that the mid-gap states created by Se vacancy are located in the vicinity of the Fermi level and thus decrease the bandgap of the bilayer $\mathrm{PtSe}_{2}$. The fact that only the samples with controlled Ar plasma treatment processes a bandgap smaller than the excitation photon energy and exhibit distinct photoresponse validates our theoretical predictions of the bandgap evolution of bilayer $\mathrm{PtSe}_{2}$ via defect engineering. The presented results indicate the importance of Se vacancy over the bandgap of $\mathrm{PtSe}_{2}$ atomic layers, suggesting that defect engineering could be an effective strategy to control the bandgap and the electronic structure of $\mathrm{PtSe}_{2}$ atomic layers.

In summary, we successfully synthesize a narrow bandgap $2 \mathrm{D}$ $\mathrm{PtSe}_{2}$ crystal and obtain large area $\mathrm{PtSe}_{2}$ with atomic layers. The 
trilayer and thicker $\mathrm{PtSe}_{2}$ are semi-metallic, while bilayer and monolayer $\mathrm{PtSe}_{2}$ are $n$-type semiconductors with indirect bandgap. Significantly, it is experimentally proved that the bandgap can be further modulated through defect engineering (e.g., varying Se vacancy) to cover the mid-IR band continuously, which is important for mid-infrared photonics and optoelectronics. As a demonstrative example, we obtain the photodetection properties of the monolayer and the bilayer $\mathrm{PtSe}_{2}$ FET devices, and find that monolayer $\mathrm{PtSe}_{2}$ FET devices are suitable for visible and near-infrared photodetectors, and bilayer $\mathrm{PtSe}_{2}$ FET devices are suitable for broadband mid-infrared photodetectors. The broadband ranges from the visible to the midinfrared along with high responsivity and fast-response speed. These results show that $\mathrm{PtSe}_{2}$ and other narrow bandgap TMCs are highly promising platforms for novel atomically thin optoelectronic applications, especially in the mid-infrared region, and they could be used to further diversify the family of 2D materials.

\section{Methods}

Experimental section. Synthesis of $\mathrm{PtSe}_{2}$ crystal flakes: The experiment setup is shown in Fig. 1c. The charges of platinum (powder, 99.9\%, Sigma-Aldrich), selenium (powder, 99.9\%, Sigma-Aldrich), red phosphorus (lump,99.9\%, SigmaAldrich), and S (powder, 99.99\%, Sigma-Aldrich) in the molar ratio of 1:2:1:3 with a total weight of $700 \mathrm{mg}$ plus $35 \mathrm{mg} \mathrm{I}$ as the transport gas were sealed in an evacuated $20 \mathrm{~cm}$ long quartz tube under vacuum at $10^{-6}$ Torr, which was placed in a three-zone furnace. Firstly, the charge pre-reacted at $900^{\circ} \mathrm{C}$ for $40 \mathrm{~h}$. Secondly, at a lower temperrature of $700^{\circ} \mathrm{C}$ for 5 days, the temperature of the reaction zone did not change with the growth zone to form a temperature gradient at which the growth of the single crystals take place, and finally, the furnace was naturally cooled down to room temperature in order to get good single crystals of $\mathrm{PtSe}_{2}$. Alternative ratios were tried, but the ratio of Pt:Se:P:S in 1:2:1:3 is optimal. In addition, the single crystals of $\mathrm{PtSe}_{2}$ cannot be obtained without $\mathrm{P}$ and $\mathrm{S}$, which are necessary to obtain the high-quality single crystals and play a catalytic role in the growing process of $\mathrm{PtSe}_{2}$ single crystals.

Material characterization. Raman data are collected at room temperature using $532 \mathrm{~nm}$ laser as the excitation source (WITec alpha 300 RAS Raman system). The sample size, thickness, morphologies, and atomic structures were conducted by optical microscopy (Olympus BX51), AFM, TEM, and high-resolution STEM.

Electrical and optical characterization. The electrical characteristics are examined by a semiconductor analyzer (Agilent, B1500A). The photoresponsivity measurement is performed in a digital deep-level transient spectroscopy (BIORAD) system with different lasers to illuminate the whole device with the semiconductor diode $(632 \mathrm{~nm})$, fiber laser $(1.47 \mu \mathrm{m}, \mathrm{FC}-\mathrm{W}-1470)$, and quantum cascade laser (10 $\mu \mathrm{m}$ QCL, DAYLIGHT TLS-41105). The lasers are focused by a parabolic mirror followed by a mechanical chopper with a frequency controller. The noise spectra is acquired by a spectrum analyzer (Keysight M9018A, with a measuring bandwidth of $10 \mathrm{kHz}$ ) with source-drain bias supplied by Agilent $1500 \mathrm{~A}$ at ambient conditions.

Data availability. The data that support the plots with this paper and other findings of this study are available from the corresponding author upon reasonable request.

Received: 25 July 2017 Accepted: 19 March 2018

Published online: 18 April 2018

\section{References}

1. Michel, J., Liu, J. \& Kimerling, L. C. High-performance Ge-on-Si photodetectors. Nat. Photon. 4, 527-534 (2010).

2. Martyniuk, M, Dell, J. W. \& Faraone, L. Mercury Cadmium Telluride: Growth, Properties and Applications (John Wiley \& Sons, Chichester, 2010).

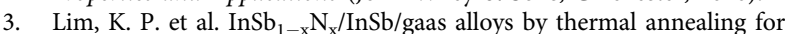
midinfrared photodetection. Appl. Phys. Lett. 97, 221112 (2010).

4. Haddadi, A. et al. InAs/InAs ${ }_{1-\mathrm{x}} \mathrm{Sb} \mathrm{b}_{\mathrm{x}}$ type-II superlattices for high performance long wavelength infrared detection. Appl. Phys. Lett. 105, 121104 (2014).

5. Sai-Halasz, G. A., Tsu, R. \& Esaki, L. A new semiconductor superlattice. Appl. Phys. Lett. 30, 651-653 (1977).

6. Bonaccorso, F. et al. Graphene, related two-dimensional crystals, and hybrid systems for energy conversion and storage. Science 347, 10 (2015).
7. George, P. A. et al. Ultrafast optical-pump terahertz-probe spectroscopy of the carrier relaxation and recombination dynamics in epitaxial graphene. Nano Lett. 8, 4248-4251 (2008).

8. Sols, F., Guinea, F. \& Neto, A. H. C. Coulomb blockade in graphene nanoribbons. Phys. Rev. Lett. 99, 166803 (2007).

9. $\mathrm{Li}, \mathrm{X}$. et al. Chemically derived, ultrasmooth graphene nanoribbon semiconductors. Science 319, 1229-1232 (2008).

10. Wei, D. et al. Controllable unzipping for intramolecular junctions of graphene nanoribbons and single-walled carbon nanotubes. Nat. Commun. 4, 1374 (2013).

11. Guo, Q. et al. Black phosphorus mid-infrared photodetectors with high gain Nano Lett. 16, 4648-4655 (2016).

12. Chen, X. et al. Widely tunable black phosphorus mid-infrared photodetector. Nat. Commun. 8, 1672 (2017).

13. Sun, Z., Martinez, A. \& Wang, F. Optical modulators with 2D layered materials. Nat. Photon. 10, 227-238 (2016).

14. Wang, Q. H. et al. Electronics and optoelectronics of two-dimensional transition metal dichalcogenides. Nat. Nanotechnol. 7, 699-712 (2012).

15. Mak, K. F. \& Shan, J. Photonics and optoelectronics of 2D semiconductor transition metal dichalcogenides. Nat. Photon. 10, 216-226 (2016).

16. Guo, G. Y. \& Liang, W. Y. The electronic structures of platinum dichalcogenides: $\mathrm{PtS}_{2}, \mathrm{PtSe}_{2}$ and $\mathrm{PtTe}_{2}$. J. Phys. C: Solid State Phys. 19, 995 (1986).

17. Mingzhe, Y. et al. High quality atomically thin $\mathrm{PtSe}_{2}$ films grown by molecular beam epitaxy. 2D Mater. 4, 045015 (2017).

18. Ferrari, A. C. et al. Raman spectrum of graphene and graphene layers. Phys. Rev. Lett. 97, 187401 (2006).

19. Wang, Y. et al. Monolayer $\mathrm{PtSe}_{2}$, a new semiconducting transition-metaldichalcogenide, epitaxially grown by direct selenization of pt. Nano Lett. 15, 4013-4018 (2015).

20. Lei, S. et al. Evolution of the electronic band structure and efficient photodetection in atomic layers of inse. ACS Nano 8, 1263-1272 (2014).

21. Lei, S. et al. Atomically layered InSe avalanche photodetector. Nano Lett. 15, 3048-3055 (2015).

22. Lin, M. et al. Controlled growth of atomically thin $\mathrm{In}_{2} \mathrm{Se}_{3}$ flakes by van der waals epitaxy. J. Am. Chem. Soc. 135, 13274-13277 (2013).

23. Jacobs-Gedrim, R. B. et al. Extraordinary photoresponse in two-dimensional $\mathrm{In}_{2} \mathrm{Se}_{3}$ nanosheets. ACS Nano 8, 514-521 (2014).

24. Choi, W. et al. High-detectivity multilayer $\mathrm{MoS}_{2}$ phototransistors with spectral response from ultraviolet to infrared. Adv. Mater. 24, 5832-5836 (2012).

25. Island, J. O. et al. Ultrahigh photoresponse of few-layer $\mathrm{TiS}_{3}$ nanoribbon transistors. Adv. Opt. Mater. 2, 641-645 (2014).

26. Lopez-Sanchez, O. et al. Ultrasensitive photodetectors based on monolayer $\mathrm{MoS}_{2}$. Nat. Nanotechnol. 8, 497-501 (2013).

27. Yin, Z. et al. Single-layer $\mathrm{MoS}_{2}$ phototransistors. ACS Nano 6, 74-80 (2011).

28. Buscema, M. et al. Fast and broadband photoresponse of few-layer black phosphorus field-effect transistors. Nano Lett. 14, 3347-3352 (2014).

29. Buscema, M. et al. Photovoltaic effect in few-layer black phosphorus pn junctions defined by local electrostatic gating. Nat. Commun. 5, 4651 (2014).

30. Wu, J. et al. Colossal ultraviolet photoresponsivity of few-layer black phosphorus. ACS Nano 9, 8070-8077 (2015).

31. Youngblood, N., Chen, C., Koester, S. J. \& Li, M. Waveguide-integrated black phosphorus photodetector with high responsivity and low dark current. Nat. Photon. 9, 247-252 (2015).

32. Yu, X., Zhang, S., Zeng, H. \& Wang, Q. J. Lateral black phosphorene $\mathrm{p}-\mathrm{n}$ junctions formed via chemical doping for high performance near-infrared photodetector. Nano Energy 25, 34-41 (2016).

33. Balandin, A. A. Low-frequency $1 / f$ noise in graphene devices. Nat. Nanotechnol. 8, 549-555 (2013).

34. Zhang, Y. et al. Broadband high photoresponse from pure monolayer graphene photodetector. Nat. Commun. 4, 1811 (2013).

35. Son, J. G. et al. Sub-10 $\mathrm{nm}$ graphene nanoribbon array field-effect transistors fabricated by block copolymer lithography. Adv. Mater. 25, 4723-4728 (2013).

36. Yu, X. et al. A high performance, visible to mid-infrared photodetector based on graphene nanoribbons passivated with $\mathrm{HfO}_{2}$. Nanoscale 8, 327-332 (2016).

37. Jena, D. \& Konar, A. Enhancement of carrier mobility in semiconductor nanostructures by dielectric engineering. Phys. Rev. Lett. 98, 136805 (2007).

38. Amani, M. et al. Mid-wave infrared photoconductors based on black phosphorus-arsenic alloys. ACS Nano 11, 11724-11731 (2017).

39. Mueller, T., Xia, F. \& Avouris, P. Graphene photodetectors for high-speed optical communications. Nat. Photon. 4, 297-301 (2010).

40. Xia, F. et al. Ultrafast graphene photodetector. Nat. Nanotechnol. 4, 839-843 (2009).

41. Gabor, N. M. et al. Hot carrier-assisted intrinsic photoresponse in graphene. Science 334, 648-652 (2011).

42. Freitag, M., Low, T. \& Avouris, P. Increased responsivity of suspended graphene photodetectors. Nano Lett. 13, 1644-1648 (2013). 
43. Chitara, B., Panchakarla, L. S., Krupanidhi, S. B. \& Rao, C. N. R. Infrared photodetectors based on reduced graphene oxide and graphene nanoribbons. Adv. Mater. 23, 5419-5424 (2011).

44. Perea-López, N. et al. Photosensor device based on few-layered $\mathrm{WS}_{2}$ films. Adv. Funct. Mater. 23, 5511-5517 (2013).

45. Yao, J. D., Zheng, Z. Q., Shao, J. M. \& Yang, G. W. Stable, highly-responsive and broadband photodetection based on large-area multilayered $\mathrm{WS}_{2}$ films grown by pulsed-laser deposition. Nanoscale 7, 14974-14981 (2015).

46. Son, Y. et al. Observation of switchable photoresponse of a monolayer $\mathrm{WSe}_{2}-\mathrm{MoS}_{2}$ lateral heterostructure via photocurrent spectral atomic force microscopic imaging. Nano Lett. 16, 3571-3577 (2016).

47. Jung, C. et al. Highly crystalline cvd-grown multilayer $\mathrm{MoSe}_{2}$ thin film transistor for fast photodetector. Sci. Rep. 5, 15313 (2015).

48. Yu, P. et al. Fast photoresponse from $1 T$ tin diselenide atomic layers. $A d v$. Funct. Mater. 26, 137-145 (2016).

49. $\mathrm{Su}, \mathrm{G}$. et al. Chemical vapor deposition of thin crystals of layered semiconductor $\mathrm{SnS}_{2}$ for fast photodetection application. Nano Lett. 15, 506-513 (2015).

50. Lei, S. et al. Ternary $\mathrm{CuIn}_{7} \mathrm{Se}_{11}$ : towards ultra-thin layered photodetectors and photovoltaic devices. Adv. Mater. 26, 7666-7672 (2014).

51. Long, $\mathrm{M}$. et al. Room temperature high-detectivity mid-infrared photodetectors based on black arsenic phosphorus. Sci. Adv. 3, e1700589 (2017).

\section{Acknowledgements}

This work is supported by funding from Ministry of Education, Singapore grant (MOE2016-T2-1-128, MOE2015-T2-2-007, MOE2015-T2-2-153, MOE Tier 1 RG164/ 15) and National Research Foundation, Competitive Research Program (NRF-CRP182017-02). This work is also supported by the Singapore National Research Foundation under NRF RF Award No. NRF-RF2013-08, the start-up funding from Nanyang Technological University (M4081137.070) and NSFC (61704082) and Natural Science Foundation of Jiangsu Province (BK20170851). Work at National University of Singapore is supported by the National Research Foundation, Prime Minister's Office, Singapore under its NRF fellowship (NRF Award No. NRF-NRFF2013-03) and benefited from the high-performance computing center of Center for Advanced 2D Materials and Graphene Research Center. The authors also acknowledge financial support from and National Natural Science Foundation of China (61704082) and Natural Science Foundation of Jiangsu Province (BK20170851) and the CAS Pioneer Hundred Talents Program

\section{Author contributions}

X.Y. and P.Y. conceived the experiments. P.Y. synthesized the crystals. X.Y. fabricated the photodetector devices and performed material characterizations and optoelectrical measurements. D.W. and B.S. performed the first-principles calculations. W.Z. conducted the TEM and STEM characterizations. Z.L. and Q.J.W. supervised the project. All authors discussed the results and commented on the manuscript.

\section{Additional information}

Supplementary Information accompanies this paper at https://doi.org/10.1038/s41467018-03935-0.

Competing interests: The authors declare no competing interests.

Reprints and permission information is available online at http://npg.nature.com/ reprintsandpermissions/

Publisher's note: Springer Nature remains neutral with regard to jurisdictional claims in published maps and institutional affiliations.

(c) (i) Open Access This article is licensed under a Creative Commons Attribution 4.0 International License, which permits use, sharing, adaptation, distribution and reproduction in any medium or format, as long as you give appropriate credit to the original author(s) and the source, provide a link to the Creative Commons license, and indicate if changes were made. The images or other third party material in this article are included in the article's Creative Commons license, unless indicated otherwise in a credit line to the material. If material is not included in the article's Creative Commons license and your intended use is not permitted by statutory regulation or exceeds the permitted use, you will need to obtain permission directly from the copyright holder. To view a copy of this license, visit http://creativecommons.org/ licenses/by/4.0/.

(C) The Author(s) 2018 